\section{Atenção primária, atenção básica e saúde da família: sinergias e singularidades do contexto brasileiro}

\author{
Primary health care, basic health care, and family \\ health program: synergies and singularities \\ in the Brazilian context
}

Célia Regina Rodrigues Gil 1

\section{Introdução}

This article analyzes the concepts of primary health care, basic health care, and family health care as used in official documents by the Brazilian Ministry of Health, final reports of the $\mathrm{Na}$ tional Conferences on Health and Human Resources, the Basic Operational Ruling on $\mathrm{Hu}$ man Resources, and texts accessed on-line by BIREME. The data analysis, through double-entry matrices, showed a lack of these references in Brazilian health policy formulation and implementation. Basic Operational Ruling 96 (NOB/96) plays a distinct role in this regard; the national conference reports show an important gap in this debate, and most of the published articles present the concepts of primary care and basic care with the meaning of health unit or local service. Articles on the Family Health Program refer to it more as a program than a strategy, and the articles analyzing such concepts show the influence of rationalities underlying the different strategies for organization of health services in the Brazilian scenario, namely Health Surveillance and Programmatic Actions in Health and in Defense of Life.

Primary Health Care; Family Health Program; Health Policy
Este artigo analisa os conceitos de Atenção Primária, Atenção Básica e Saúde da Família, presentes no cenário da reforma sanitária brasileira, no que se refere ao modelo de atenção à saúde e organização dos serviços municipais que, na trajetória da descentralização foram sendo incorporados paulatinamente em nosso contexto. As reflexões em torno do legado da Atenção Primária à Saúde e sua estratégia dos cuidados primários ainda hoje suscitam debates entre sujeitos e atores sociais envolvidos nos rumos da Política Nacional de Saúde. No período anterior à criação do Sistema Único de Saúde (SUS), a Atenção Primária à Saúde representava um marco referencial para a organização dos serviços numa lógica que tinha como proposta ser uma das principais alternativas de mudança do modelo assistencial. Após sua criação e o desenvolvimento de seus mecanismos financeiros e operacionais, cada vez tem sido mais freqüente o uso do conceito Atenção Básica como referência aos serviços municipais. Nos últimos anos, vimos crescer no cenário brasileiro o Programa Saúde da Família (PSF), que vivificou este debate ao explicitar a superposição destes referenciais que permeiam a organização dos sistemas locais. Há experiências municipais que fazem referência à organização do PSF na perspectiva da Atenção Primária, outros o fazem na da Atenção Básica, sendo 
que estes termos são utilizados ora como sinônimos, ora como contraposição.

Reconhecendo o papel que o contexto internacional exerce nas definições das políticas de saúde e seu poder de influência na determinação de modelos e práticas assistenciais, observa-se que a criação das agências internacionais 1 de sustentação do capital financeiro (Fundo Monetário Internacional - FMI, Banco Mundial) e das políticas de segurança e paz no mundo (Organização das Nações Unidas - ONU, Organização Mundial da Saúde - OMS, Fundo das Nações Unidas para a Infância - UNICEF), logo após a Segunda Grande Guerra, mudou a forma e a configuração das relações internas e externas dos países, desencadeando uma nova ordem no cenário mundial. As agências econômicas configuradas pelas instituições de Bretton Woods, de um lado, cada vez mais criam meios para assegurar a composição dos recursos financeiros das agências internacionais pela sua estrutura de poder centrada na política do voto proporcional, garantindo aos países capitalistas, detentores dos recursos, mecanismos de influência e decisão nos rumos das políticas econômicas e sociais dos diferentes países, principalmente nos do hemisfério sul. Por outro lado, as agências internacionais ligadas às Nações Unidas, voltadas às políticas sociais, vêm exercendo importante papel no sentido de viabilizar a consecução de políticas mais equânimes para os povos e entre eles, mediando conflitos de interesses entre financiamento e necessidades sociais dos países 1,2 .

A Conferência Internacional de Alma Ata, realizada em 1978, pela OMS e UNICEF, é exemplo 1,3 de um evento internacional que representou um marco de influência nos debates sobre os rumos das políticas de saúde no mundo, reafirmando a saúde como direito humano fundamental ${ }^{3}$. Em que pese ter sido realizada num contexto acirrado da crise monetária internacional, ela continuou influenciando mudanças no cenário mundial durante toda a década de 1980 , considerada a dos anos do ajuste fiscal 1,2,4.

Ressalta-se que, mesmo antes dessa crise, muitos países já vinham enfrentando dificuldades no setor decorrentes da crescente elevação dos gastos em saúde, levando à necessidade de reformas setoriais em muitos deles, configurando um cenário de "movimento universal em prol da reforma dos sistemas de saúde" 5 (p. 27), que abarca desde a necessidade de mudanças do modelo assistencial até a melhor alocação, racionalização e utilização dos recursos 5. Aponta-se, como um dos importantes vetores dessa crise, a influência do modelo médico hegemônico 5,6,7,8 que, se por um lado tem sido altamente eficiente na elevação destes gastos, por outro, tem se mostrado insuficiente quanto ao impacto na melhoria da qualidade de vida e saúde da população e otimização dos serviços. Este modelo é tido como um dos fatores da ineficácia e encarecimento do sistema e, no caso brasileiro, aponta para a necessidade de se dar continuidade ao processo da reforma sanitária brasileira em direção a uma nova etapa 5,8, a chamada de "a reforma da reforma".

Países como Canadá e Espanha que tiveram esse conjunto de fatores agravando a crise setorial, têm encontrado, entre as estratégias para superá-la, a adoção da atenção primária à saúde como marco referencial a partir do qual tem se dado a reestruturação do setor e organização dos serviços 5,9. Entretanto, por ter sido realizada e difundida em um período e contexto histórico de fortalecimento das propostas de ajuste fiscal do FMI e Banco Mundial, nos países latino-americanos, pouca atenção foi dada ao fato de que os princípios defendidos na Conferência Internacional de Alma Ata podem ter representado um dos últimos esforços de criação de um padrão internacional de pactuação no qual os países em desenvolvimento tiveram voz e representatividade em um fórum internacional 1,2 .

No caso brasileiro, o referencial proposto em Alma Ata inspirou as primeiras experiências de implantação dos serviços municipais de saúde 10 no final da década de 1970 e início de 1980, e trouxe aportes conceituais e práticos para a organização dos mesmos. Porém, no transcorrer do tempo e com o desenvolvimento do SUS, este referencial parece perder gradativamente sua potência sendo, cada vez mais, substituído pela concepção da Atenção Básica à Saúde e, em meados da década de 1990, com a implantação do PSF e dos incentivos financeiros específicos dirigidos aos municípios, em especial o Piso da Atenção Básica 11 em 1998 que resultou no fortalecimento da Atenção Básica e do PSF no âmbito municipal, percebe-se uma intensificação deste movimento.

A pergunta que emerge é se há diferentes compreensões acerca dessas concepções e se as mesmas podem ser entendidas como sinônimos no contexto brasileiro. Questiona-se, ainda, se a Saúde da Família sofre influências decorrentes da superposição desses conceitos ou diferentes interpretações sobre Atenção Primária à Saúde, uma vez que há referências à Saúde da Família ora como proposta estruturante para a organização da Atenção Básica, ora como da Atenção Primária, e ainda, como mais um dos programas da rede básica de serviços. Procurando elucidar tais questões e melhor 
compreender o cenário de implementação do PSF enquanto política pública, buscou-se, neste estudo, evidenciar como vem se dando este debate no âmbito da reforma sanitária e desenvolvimento do SUS, por meio da análise de documentos oficiais do Ministério da Saúde (MS) e revisão de literatura.

\section{Estratégia metodológica}

Considerando que a organização dos serviços não acontece ao acaso e que as práticas profissionais, no seu interior, ocorrem articuladas a um corpo de idéias, a um conjunto de ações coordenadas que configuram um determinado processo de trabalho em saúde estruturado pela forma como os serviços são organizados, geridos e financiados, julgou-se importante, nesta discussão, conhecer o posicionamento do MS, por ter sido ele o principal órgão formulador e financiador das políticas de saúde. Buscaram-se elementos que permitissem identificar a racionalidade que tem pautado a formulação das propostas de estruturação, organização e financiamento dos serviços básicos priorizados pelo MS. Do mesmo modo, julgou-se importante conhecer o pensamento de diferentes atores sociais ligados aos SUS sobre essa temática, uma vez que estes têm poder de influência no processo de implementação (ou não) das políticas de saúde e, conseqüentemente, na mudança do modelo de atenção.

Partindo dessas premissas, foram analisados documentos oficiais do MS, no período de 1990 a 2005, nos quais se procurou evidenciar como esse órgão tem apresentado tais concepções e se as mesmas têm sido orientadoras das propostas de desenvolvimento das ações básicas de saúde no âmbito do SUS, entendendo desenvolvimento como formulação e implementação das políticas de saúde.

Para tanto, fez-se uma revisão dos principais documentos normativos do SUS: as Normas Operacionais Básicas (NOB) 12,13, Norma Operacional da Assistência à Saúde (NOAS) 14, Leis Orgânicas da Saúde 15,16 e dos principais documentos orientadores da Atenção Básica, em especial os mais difundidos aos municípios 17,18, 19,20. Para complementar o estudo, fez-se um levantamento dos relatórios finais da 7ạ a 12a Conferência Nacional de Saúde (CNS) 21,22,23,24, 25,26, das Conferências Nacionais de Recursos Humanos em Saúde (CNRHS) 27,28 e da Norma Operacional Básica de Recursos Humanos para o SUS (NOB/RH/SUS) 29, considerando-as representativas do debate democrático entre academia, gestores, profissionais e usuários do SUS, e com papel importante na proposição da agenda político-estratégica da saúde.

Concomitantemente, fez-se uma revisão de literatura acessando a base de dados da BIREME (Biblioteca Virtual em Saúde - BVS: http:/ / www. saudepublica.bvs.br) a partir dos descritores e palavras-chave "Atenção Primária à Saúde", "Atenção Básica à Saúde" e "Programa Saúde da Família” buscando informações acerca das possíveis interpretações dadas pelos diferentes autores e atores sociais a estes conceitos. Foram selecionados os textos completos apresentados na SciELO por ser possível ter acesso integral aos artigos e por considerá-la fonte da qual muitos pesquisadores e estudiosos fazem uso. Nesses documentos identificou-se o contexto em que apareceram ou foram citados tais conceitos-chave, objetos do estudo. Os dados encontrados foram sistematizados por meio da utilização de matrizes de dupla entrada, cruzando-se características e autores/documentos nos quais as mesmas foram encontradas. Isso posto, procedeu-se a análise final complementando-a com outras referências pertinentes.

\section{Resultados}

Na NOB-93 12 e nas Leis Orgânicas 15,16 não há indicativos sobre modelos de atenção à saúde e não foram encontradas referências sobre ações específicas que deveriam estar asseguradas pelo SUS nos diferentes níveis de complexidade do sistema. Explicitamente citadas estão as ações de vigilância sanitária e epidemiológica, saúde do trabalhador, alimentação e nutrição e saneamento básico. Da mesma forma, não se observa qualquer recomendação sobre organização dos cuidados primários ou serviços básicos de saúde como responsabilidade do primeiro nível de assistência. Na NOB96 é que estão as primeiras citações sobre a mudança do modelo de atenção à saúde, já destacando o PSF como opção.

Considerando que as NOBs têm sido mecanismos de viabilização do SUS e que cada uma delas imprimiu concepções e ritmos distintos ao processo de descentralização do SUS 30,31, nos parece que a NOB-96 inaugurou uma nova retórica na organização dos serviços municipais, conforme aponta a Tabela 1 .

Nos relatórios finais das CNS 21,22,23,24,25,26 percebe-se que nas duas últimas conferências a discussão sobre modelo assistencial esteve mais presente e, em ambas, na perspectiva da Atenção Básica. Observa-se que na 12a CNS, o PSF ganha um espaço maior nas discussões, embora perdurando questionamentos em não 
Referências sobre Atenção Primária, Atenção Básica e Saúde da Família encontradas nos documentos do Ministério da Saúde, 1990 a 2005.

\begin{tabular}{|c|c|c|}
\hline Documentos & Ano & Referências encontradas \\
\hline Lei n. 8.080 e Lei n. 8.142 & 1990 & Nenhuma referência foi encontrada. \\
\hline NOB-91 e NOB-93 & $1991 / 1993$ & Idem. \\
\hline NOB-93 & 1993 & Idem. \\
\hline NOB-96 & 1998 & $\begin{array}{l}\text { Cita Saúde da Família. Aponta para a reordenação do modelo de atenção centrada não } \\
\text { apenas no financiamento, mas também nos resultados esperados, destaca a importância de } \\
\text { vínculo entre serviços e usuários, privilegiando os núcleos familiares e comunidade; ressalta } \\
\text { a integralidade das ações e necessidade de incorporar no modelo clínico, o modelo } \\
\text { epidemiológico. Explicita financiamento ao PSF e Programa de Agentes Comunitários de } \\
\text { Saúde. Utiliza os termos serviços básicos e ações básicas, mas não fala explicitamente em } \\
\text { Atenção Básica. Não faz referência à Atenção Primária à Saúde e nível primário de atenção. }\end{array}$ \\
\hline $\begin{array}{l}\text { Saúde da Família no Brasil: } \\
\text { Linhas Estratégicas para } \\
\text { o Quadriênio 1999/2002 }\end{array}$ & 1999 & $\begin{array}{l}\text { Nenhuma referência sobre Atenção Primária à Saúde ou similar. No documento, trata a } \\
\text { Atenção Básica na perspectiva da organização dos serviços de saúde do âmbito municipal. }\end{array}$ \\
\hline Manual da Atenção Básica & 1999 & $\begin{array}{l}\text { Nenhuma referência sobre Atenção Primária ou similar. Propõe ações para orientar } \\
\text { a organização da Atenção Básica nos municípios. }\end{array}$ \\
\hline $\begin{array}{l}\text { O Trabalho do Agente } \\
\text { Comunitário de Saúde }\end{array}$ & 2000 & $\begin{array}{l}\text { Resgata e contextualiza historicamente a Conferência de Alma Ata e os conceitos de Atenção } \\
\text { Primária e cuidados primários de saúde; a Carta de Otawa e a Promoção da Saúde, } \\
\text { apresentando sucintamente as Conferências Internacionais de Promoção da Saúde; a 8a CNS, } \\
\text { a criação do SUS, a descentralização e participação e, ao falar da Atenção Básica à Saúde, } \\
\text { os termos atenção primária, assistência primária, açães do primeiro nível de assistência e } \\
\text { atenção básica são tratados como sinônimos. Ao discorrer sobre o Programa de Agentes } \\
\text { Comunitários de Saúde e PSF predomina a fala sobre a Atenção Básica. }\end{array}$ \\
\hline $\begin{array}{l}\text { Guia Prático de Implantação } \\
\text { do Programa Saúde } \\
\text { da Família (PSF) }\end{array}$ & 2001 & $\begin{array}{l}\text { Nenhuma citação sobre atenção primária ou similar. Faz referência aos serviços básicos } \\
\text { de saúde e à rede básica como integrante da rede de saúde. Em todo o documento, trata } \\
\text { a Atenção Básica na perspectiva da organização dos serviços de saúde no município. }\end{array}$ \\
\hline $\begin{array}{l}\text { Regionalização da Assistência } \\
\text { à Saúde: NOAS/SUS 01/01 }\end{array}$ & 2002 & $\begin{array}{l}\text { Nenhuma referência sobre atenção primária ou similar. O documento utiliza o conceito } \\
\text { de Atenção Básica na perspectiva de organização dos serviços, trata especificamente } \\
\text { da proposta de estruturação da rede de serviços. Em todo o documento, não faz uma } \\
\text { referência sequer ao PSF. }\end{array}$ \\
\hline
\end{tabular}

torná-lo uma proposta minimalista de atenção à saúde. A Tabela 2 apresenta uma síntese das principais idéias contidas nos documentos das conferências.

Nos relatórios finais das CNRHS 27,28 e no documento da NOB/RH/SUS 29, nenhuma referência específica sobre tais concepções foram encontradas.

Na pesquisa on-line da BVS, no ícone “Terminologia em Saúde", foram pesquisados os descritores "Atenção Primária à Saúde", "Atenção Básica à Saúde" e "Programa Saúde da Família" e foi observado que o descritor "Atenção Primária à Saúde", ao estabelecer os sinônimos para cuidados primários de saúde, deixa margem à interpretação reducionista da saúde, pois aparece li- gado às concepções de pacote básico de serviços, cesta básica de saúde, entre outros, indo ao encontro das críticas sobre as idéias difundidas pelas agências internacionais de racionalização do financiamento em saúde. Nada foi encontrado na busca ao descritor "Atenção Básica à Saúde", o que reforça a impressão de que esta concepção é recente. O descritor "Programa Saúde da Família" apresenta uma definição ampla que explicita seu propósito de reorganização da prática de atenção à saúde e a substituição do modelo tradicional, a composição das equipes e os parâmetros populacionais. Sintetizamos os resultados deste detalhamento na Figura 1.

Acessando a opção de busca "Brasil/Saúde Pública”, foram encontradas indexações para 
Referências sobre Atenção Primária, Atenção Básica e Saúde da Família presentes nos relatórios finais das Conferências Nacionais de Saúde, 1980 a 2005.

\begin{tabular}{|c|c|}
\hline Conferências nacionais & Referências encontradas \\
\hline $\begin{array}{l}\text { VII } \\
\text { (Março/1980) } \\
\text { Ministro: Waldir Arcoverde } \\
\text { Tema central } \\
\text { Extensão das ações de saúde através } \\
\text { dos serviços básicos. }\end{array}$ & $\begin{array}{l}\text { Ministro destaca a intersetorialidade e a Atenção Primária à saúde como caminho para mudar } \\
\text { a saúde no Brasil. Cita ações básicas e assistência básica de saúde. Diretor geral da OMS } \\
\text { enfatiza a Atenção Primária à Saúde e a Conferência de Alma Ata. O presidente destaca } \\
\text { a integração e coordenação entre ministérios da Saúde, da Previdência e da Educação } \\
\text { e a importância das ações básicas de saúde (ações de prevenção e diagnóstico precoce). } \\
\text { No relatório final, nas recomendações dos } 11 \text { subtemas discutidos, aparece o conceito de } \\
\text { serviços básicos como avanço e alguns fazem menção à Atenção Primária à Saúde como nível } \\
\text { de organização dos serviços; há apontamentos para a descentralização do sistema, } \\
\text { municipalização e integração da rede básica com os serviços secundários e terciários. }\end{array}$ \\
\hline $\begin{array}{l}\text { VIII } \\
\text { (Março/1986) } \\
\text { Ministro: Roberto Santos } \\
\text { Temas centrais } \\
\text { Saúde como direito inerente à personalidade } \\
\text { e à cidadania; reformulação do sistema } \\
\text { nacional de saúde. } \\
\text { Financiamento setorial. }\end{array}$ & $\begin{array}{l}\text { Ministro faz pequena referência aos cuidados primários e grande destaque às ações } \\
\text { integradas de saúde, à regionalização, descentralização e municipalização dos serviços. } \\
\text { Diretor Geral da OMS faz referência ao marco "saúde para todos" e ressalta os princípios } \\
\text { da eqüidade e universalização. Não faz referência à Atenção Primária à Saúde ou similar. } \\
\text { O presidente faz referências às desigualdades sociais no Brasil, aborda a dificuldade de } \\
\text { acesso à saúde e destaca a racionalidade administrativa mediante descentralização dos } \\
\text { serviços. O relatório final ressalta: tema } 1 \text { - a importância de uma nova Constituição; tema } \\
2 \text { - a necessidade de criação do SUS e seus princípios norteadores e a municipalização; tema } \\
3 \text { - a criação dos Fundos de Saúde. Não há referências sobre Atenção Primária à Saúde, } \\
\text { atenção básica ou assistência básica. Esta CNS centrou-se no sistema nacional de saúde. }\end{array}$ \\
\hline $\begin{array}{l}\text { IX } \\
\text { (Agosto/1992) } \\
\text { Ministro: Adib Jatene } \\
\text { Tema central } \\
\text { Saúde: municipalização é o caminho }\end{array}$ & $\begin{array}{l}\text { Faz recomendações sobre mudança do modelo assistencial, apontando para as bases } \\
\text { epidemiológicas; fala em práticas diferenciadas segundo realidades locais, fala em } \\
\text { complexidade crescente dos serviços e da atenção integral à saúde. Nenhuma citação sobre } \\
\text { a Atenção Primária à Saúde ou sobre Atenção Básica em Saúde. No relatório final não } \\
\text { constam os discursos das autoridades. }\end{array}$ \\
\hline $\begin{array}{l}\text { X } \\
\text { (Setembro/1996) } \\
\text { Ministro: Adib Jatene } \\
\text { Tema central } \\
\text { SUS: construindo um modelo de atenção } \\
\text { à saúde para a qualidade de vida }\end{array}$ & $\begin{array}{l}\text { Ministro recupera as CNS anteriores, menciona a influência de Alma Ata nos anos } 80 \\
\text { e do slogan "Saúde para todos no ano 2000". } \\
\text { Relatório final: várias citações sobre atenção integral à saúde; repúdio à cesta básica proposta } \\
\text { pelo Banco Mundial; ênfase nas proposições de concurso público para ingresso nos serviços } \\
\text { públicos. PSF e Programa de Agentes Comunitários de Saúde aparecem em item especial, } \\
\text { destacando a necessidade de debate com os Conselhos de Saúde para definir a necessidade } \\
\text { ou não de implantação destes programas que não deve ser feita de forma centralizada } \\
\text { e vertical. A NOB } 96 \text { é bastante debatida nesta CNS Nenhuma citação sobre Atenção } \\
\text { Primária à Saúde e Atenção Básica ou similares. }\end{array}$ \\
\hline $\begin{array}{l}\text { XI } \\
\text { (Dezembro/ 2000) } \\
\text { Ministro: José Serra } \\
\text { Tema central } \\
\text { Efetivando o SUS: acesso, qualidade } \\
\text { e humanização na atenção à saúde, } \\
\text { com controle social. }\end{array}$ & $\begin{array}{l}\text { No Relatório Final desta Conferência não constam os discursos das autoridades oficiais. } \\
\text { Nenhuma citação sobre APS ou cuidados primários de saúde. Encontram-se muitas } \\
\text { referências sobre Atenção Básica. Aparecem questionamentos e divergências sobre o PSF } \\
\text { como estratégia de reorganização da Atenção Básica e preocupações quanto ao PSF } \\
\text { tornar-se modelo de saúde pobre para os pobres. }\end{array}$ \\
\hline $\begin{array}{l}\text { XII } \\
\text { (Dezembro/2003) } \\
\text { Ministro: Humberto Costa } \\
\text { Tema central } \\
\text { Saúde um direito de todos e um dever do Estado. } \\
\text { A saúde que temos, o SUS que queremos. }\end{array}$ & $\begin{array}{l}\text { No eixo temático "A organização da Atenção à Saúde" coloca-se, logo no início, que } \\
\text { a mudança do modelo de atenção à saúde é hoje o principal desafio do SUS e aponta, } \\
\text { nas diretrizes gerais, a recomendação de ampliação da cobertura dos serviços em todos } \\
\text { os níveis de complexidade, com ênfase na Atenção Básica, priorizando a estratégia Saúde } \\
\text { da Família e outras semelhantes que possibilitem a mudança de orientação do modelo de } \\
\text { atenção. Apresenta um item específico sobre Atenção Básica e Saúde da Família com } \\
\text { recomendaçães de criação e fixação de incentivos técnicos e financeiros nas três esferas } \\
\text { de governo para implementação da Atenção Básica a partir da Saúde da Família, e criação } \\
\text { de equipes de referência e serviços de apoio. Nenhuma referência à Atenção Primária } \\
\text { à Saúde ou similar, faz sempre referência à atenção básica. }\end{array}$ \\
\hline
\end{tabular}


Figura 1

Descritores encontrados na Biblioteca Virtual em Saúde (BVS) e seus respectivos descritores em português.

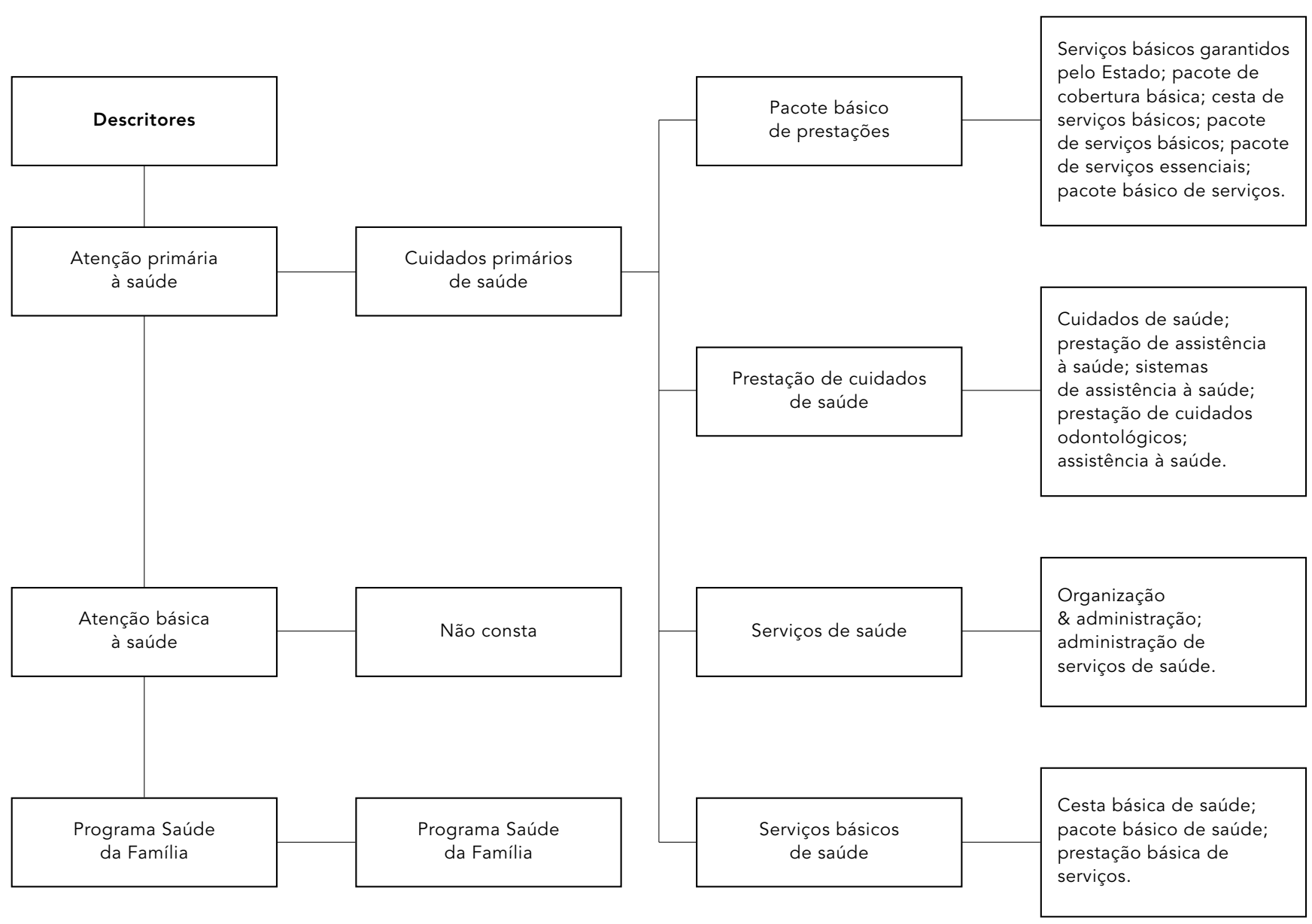

as três palavras-chave, sendo 16.844 sobre Atenção Primária à Saúde, 160 sobre Atenção Básica à Saúde - ambas concentradas no MEDLINE e 772 sobre PSF, concentradas no LILACS. Na SciELO, 50 textos completos sobre Atenção Primária à Saúde, 48 sobre Atenção Básica à Saúde e 59 sobre PSF foram encontrados e, destes, 26 foram excluídos por serem repetidos, restando 131 artigos que foram lidos e analisados visando a apreender o contexto em que tais conceitos foram apresentados pelos autores. A Tabela 3 demonstra como o total de artigos está disponibilizado ao público.

A literatura pesquisada mostra que a produção científica envolvendo esse tema está disseminada pelos mais conceituados periódicos do país, sendo os três primeiros avaliados com "conceito A internacional" pela Coor- denação de Aperfeiçoamento de Pessoal de Nível Superior (CAPES). A produção média nesses 14 anos foi de aproximadamente um artigo/mês. Entretanto, ao analisar os contextos dos artigos, evidencia-se que a maioria dos trabalhos sobre Atenção Primária à Saúde e Atenção Básica à Saúde trata de pesquisas e estudos de avaliação de problemas/agravos de saúde em unidades locais, ora denominadas de unidades/serviços de atenção primária, ora de unidades básicas. Menos da metade dos artigos contextualizaram suas experiências na ótica do SUS e menos de um terço problematizaram estes conceitos na perspectiva do modelo de atenção e/ou política de saúde. Isso se repete em relação aos artigos sobre PSF, mas neste subconjunto, verifica-se maior variedade de assuntos e a produção mais re- 
Textos completos disponibilizados pelo SciELO a partir das palavras-chave: Atenção Primária à Saúde, Atenção Básica e Programa Saúde da Família, por periódico. Janeiro de 1990 a março de 2005.

\begin{tabular}{lcccc}
\hline Periódico & $\begin{array}{c}\text { Atenção Primária } \\
\text { à Saúde }\end{array}$ & $\begin{array}{c}\text { Atenção Básica } \\
\text { à Saúde }\end{array}$ & $\begin{array}{c}\text { Programa Saúde } \\
\text { da Família }\end{array}$ & $\begin{array}{c}\text { Total } \\
\text { Cadernos de Saúde Pública }\end{array}$ \\
Revista de Saúde Pública & 26 & 23 & 38 & 69 \\
Revista Panamericana de Salud Pública & 8 & 9 & 2 & 6 \\
Ciência \& Saúde Coletiva & 6 & 5 & 6 & 9 \\
Revista da Associação Médica Brasileira & 1 & - & - & 3 \\
Outros & 3 & 9 & 9 & 22 \\
Total & 6 & 48 & 59 & 157 \\
\hline
\end{tabular}

Fonte: BIREME (http://www.saudepublica.bvs.br, acessado em 21/Mar/2005).

cente aponta temas mais voltados ao cuidado em saúde.

Na Tabela 4 sintetizamos as idéias centrais que permeiam os textos lidos.

\section{Discussão}

O encontrado na pesquisa on-line está de acordo com o que vimos sobre as diferentes interpretações sobre atenção primária ${ }^{5}$, que se explicam pela trajetória histórica de como esse conceito foi gerado, de como evoluiu e pela ambigüidade das definições estabelecidas em fóruns internacionais em relação ao termo Atenção Primária. Essas interpretações 5 variam de: (1) Atenção Primária à Saúde como estratégia de reordenamento do setor saúde; (2) Atenção Primária à Saúde como estratégia de organização do primeiro nível de atenção do sistema de saúde e (3) Atenção Primária à Saúde como programa com objetivos restritos e voltados especificamente à satisfação de algumas necessidades mínimas de grupos populacionais em situação de extrema pobreza e marginalidade. Nos países do terceiro mundo, predomina a interpretação da Atenção Primária à Saúde como um programa específico para os marginalizados e excluídos e materializada na proposta político-ideológica da atenção primária seletiva destinada às populações pobres 5 .

Enquanto a primeira corrente é defendida por alguns autores $5,30,32,33,34$, outros $35,36,37$ manifestam suas preocupações quanto a Atenção Primária à Saúde e o PSF serem políticas oficiais reducionistas da saúde. Estas críticas corroboram argumentações 38,39,40 quanto a inserção da Atenção Primária à Saúde em nosso contexto como proposta minimalista, voltada à focalização da saúde, e transportam estas inquietações ao PSF, questionando se o mesmo não seria o sucedâneo dos cuidados primários de saúde ou fazendo ressalvas quanto à necessidade de mudanças significativas na estruturação desses serviços. Os trabalhos que apontam inquietações quanto à Atenção Primária à Saúde ou ao PSF se constituírem em medicina simplificada 40 ou prática de saúde pobre para pobres 39 são minoritários.

Alguns autores utilizam os termos atenção primária à saúde e atenção básica à saúde como sinônimos e na perspectiva de unidades locais ou nível de assistência. Outrossim, parte dos artigos situa a atenção primária à saúde enquanto serviços de saúde do primeiro nível de atenção, indo ao encontro de sua definição como porta de entrada para o sistema, tendo, nas unidades básicas, sua instância operacional com nível próprio de atendimento 41 . A percepção que se tem ao analisar tais artigos é a de que esta concepção de Atenção Primária à Saúde predomina em nosso contexto, dado o volume de trabalhos que se utilizaram desta perspectiva em seus estudos.

Entre os que abordam esses conceitos na perspectiva de modelo, alguns discorrem sobre os diferentes modelos de atenção à saúde 35,36 ou modelos de organização tecnológica do trabalho em saúde 42 que permeiam o cenário brasileiro desde a década de 1990. São apontados como modelos: Vigilância à Saúde, Ações Programáticas de Saúde e Em Defesa da Vida. Em que pese os autores colocarem tais modelos como singulares, não encontrou-se evidências que demonstrem a predominância de um sobre outro e, a nosso ver, estas formulações podem ser comparadas a um caleidoscópio de experiências que têm, em comum, a intenciona- 
Características e contextos encontrados a partir das referências sobre Atenção Primária, Atenção Básica e Saúde da Família presentes nos textos completos, SciELO, 1990 a 2004.

\begin{tabular}{|c|c|}
\hline Palavras-chave & Características e contextos encontrados nos artigos \\
\hline Atenção Primária à Saúde & $\begin{array}{l}\text { Predominam estudos de avaliação de programas (pré-natal, puericultura, aleitamento } \\
\text { materno), problemas de saúde (diabetes, hipertensão, transtornos mentais, asma), agravos } \\
\text { (violência doméstica, aborto); questões gerenciais deste nível de prestação de serviços } \\
\text { (sistemas de informação, gerência, processo de trabalho). A Atenção Primária à Saúde } \\
\text { aparece caracterizada mais como unidade/serviço local (avaliação da prevenção da diabetes } \\
\text { em uma unidade de atenção primária) do que como serviços de âmbito municipal, estratégia } \\
\text { ou modelo de organização de serviços. Alguns artigos referem-se à Atenção Primária à Saúde } \\
\text { como açães de "prevenção primária", "educação em saúde". Dos } 42 \text { artigos, cinco se } \\
\text { caracterizaram por abordar a Atenção Primária à Saúde numa perspectiva mais ampla, } \\
\text { (modelo de atenção, estratégia de organização de sistemas municipais de saúde). }\end{array}$ \\
\hline
\end{tabular}

Atenção Básica à Saúde

Programa Saúde da Família (PSF)
Nos 42 textos sobre Atenção Básica à Saúde percebe-se maior diversificação dos temas, sendo nove estudos sobre mortalidade, dez de avaliação de programas, doenças ou agravos de saúde; quatro sobre atividades gerenciais; dois sobre acesso/territorialização; cinco sobre práticas profissionais; um sobre análise de políticas públicas e 11 sobre modelos de atenção à saúde. Quarenta porcento deles fazem referência à estratégia ou programa saúde da família. Vários fazem referência ao SUS, contextualizando-o no corpo do texto.

Nos 47 textos sobre PSF observa-se uma variedade de temas, embora os agravos/doenças predominem (21 artigos). Há produção sobre práticas profissionais, inclusão de temas como acolhimento, vínculo, processo de trabalho, trabalho em equipe (dez artigos). Há estudos sobre o trabalho do agente comunitário de saúde (três), saúde do trabalhador (dois), gerência de equipes (um), qualidade de vida e promoção (dois). Oito textos trazem reflexões sobre modelo de atenção no âmbito do SUS. Constata-se que muitos trabalhos falam no PSF como estratégia da Atenção Primária á Saúde; os termos Atenção Primária à Saúde e Atenção Básica à Saúde aparecem como sinônimos, mas nos que mais discutem o modelo assistencial o conceito de Atenção Básica à Saúde aparece com mais freqüência. lidade de produzir transformações no modelo por meio da reorganização dos serviços, reorientação das práticas e dos mecanismos de gestão. E ainda, até a expansão da Saúde da Família, não tinham alcançado visibilidade para integrar as diferentes agendas políticas do SUS (MS, Conferências de Saúde) que priorizaram, histórica e necessariamente, as discussões em torno do financiamento do sistema.

O crescimento do PSF trouxe para a centralidade dos debates a insuficiência dos arranjos organizacionais atuais e a falência das práticas profissionais e de gestão no que se refere especificamente à mudança dos paradigmas que regem o tradicional modelo de atenção à saúde. Neste sentido, pode-se considerar que o PSF avançou de programa para estratégia em parte devido ao acúmulo subjacente ao debate e experiências em torno destes referenciais. No nosso entendimento, o PSF apóia-se mais no referencial da Vigilância à Saúde 5 (organização das ações a partir do território e problemas de saúde, intersetorialidade, discriminação positiva de saúde, paradigma da produção social da saúde) que sustenta a Saúde da Família como estratégia de organização da Atenção Primária, negando as versões de que esta seria um arranjo contemporâneo da medicina simplificada, um programa, apenas medicina familiar e somente atenção materno-infantil.

Por sua vez, as Ações Programáticas de Saúde 41 (inspiradas na Programação em Saúde revisitada que trata, fundamentalmente, da discussão sobre a medicina enquanto um recurso essencialmente epidemiológico e da transição da dimensão médica individual para a coletiva) também têm seus pressupostos permeando a organização dos serviços e influenciando o PSF. Várias experiências relatadas representam buscas em torno do equilíbrio demanda espontânea versus oferta organizada, com padronização de condutas e definição de protocolos e uso da epidemiologia para a priorização das ações de saúde. Estas orientações estão incorporadas nas discussões sobre a organização dos sistemas locais e permeiam o trabalho das equipes de Saúde da Família cotidianamente.

A proposta Em Defesa da Vida 42,43 , com seu foco mais voltado aos processos de trabalho em saúde, ao investir em métodos, instrumentos de gestão e de organização do trabalho coletivo para produzir mudanças no interior do sistema 
e transformações das pessoas e de suas práticas, privilegia o acolhimento, o vínculo, o contrato e a autonomia dos sujeitos na organização progressiva do cuidado como estratégias de transformação do sistema e, como as anteriores, estas formulações estão bastante contidas nas reflexões contemporâneas e nos debates em torno da estruturação da Saúde da Família.

Portanto, há que se concordar quando Teixeira 44 afirma que essas experiências são mais estratégias do que propriamente proposta de modelos, considerando que há espaços distintos para e entre elas no âmbito do SUS. Estes referenciais privilegiam reflexões em distintas dimensões que vão desde os microprocessos relativos aos processos de trabalho em saúde, presentes no Em Defesa da Vida; os macroprocessos de organização dos serviços presentes nas Ações Programáticas de Saúde; e as macropolíticas de organização do sistema presentes na Vigilância à Saúde.

Destarte, essa análise, embora inicial, ela aponta a necessidade de um esforço sinérgico entre essas vertentes considerando que as tecnologias de gestão do sistema, a organização da rede de serviços e as transformações no processo de trabalho estão contidas nesse debate e são condições essenciais para que haja a conversão do paradigma de modelo 44 .

O diálogo proporcionado pela leitura e análise desses documentos e artigos permitiu identificar que, no contexto brasileiro, a Atenção Primária à Saúde traz consigo as seqüelas do debate travado nos anos 70, decorrentes do caráter racionalizador defendido por alguns atores das agências financeiras que preconizavam a redução do financiamento e gastos em saúde, em detrimento das reais necessidades de saúde das populações dos países periféricos.

Mesmo não tendo sido essa a racionalidade de muitos dos formuladores das políticas de saúde da época, que defendiam, inclusive, o aumento de recursos para o setor, a Atenção Primária à Saúde, por ter sido implementada num contexto no qual a expansão da cobertura veio acompanhada das propostas de contenção do financiamento 1 , teve seus pressupostos estruturantes de um novo modelo (universal, equânime, inclusivo, integral) obscurecidos pelo ideário neoliberal racionalizador (focalização, baixo custo, pacote básico, excludente). Mesmo assim, esse debate tem sido salutar por manter alerta e vigilante os diferentes segmentos sociais da reforma sanitária brasileira em relação aos rumos da política de saúde, do próprio SUS e da concepção de política setorial que se quer imprimir no país.
Vinte anos depois de Alma Ata emerge o conceito de Atenção Básica que, assim como a Atenção Primária à Saúde, sofreu influência do Banco Mundial na organização das ações de saúde. Surge igualmente num contexto de importantes ajustes econômicos, inclusive permeados por políticas de redução do papel do Estado (Estado Mínimo) que caracterizaram a década de 1990 e, apesar disso, parece ter sido mais facilmente incorporado ao cotidiano do setor saúde. Inferimos que a explicação disso reside na própria trajetória de construção do SUS, que, ao longo de sua história veio formulando instrumentos e normas para orientar a descentralização e organização do sistema, como as NOB, o PAB, as formas de habilitação do sistema etc. Esta aprendizagem institucional intensifica-se durante as negociações em torno da NOB-96, ressaltando que é a partir daí que se observa sua incorporação nos documentos oficiais do MS, Relatórios das Conferências e mesmo nos artigos pesquisados, demonstrando certa preferência por esse conceito.

Por fim, a estratégia Saúde da Família, embora tenha crescido sobremaneira no país, atravessa atualmente seu maior desafio para viabilizar-se como estratégia estruturante dos sistemas municipais, que é sua consolidação nos grandes centros urbanos. Vive, com isso, um paradoxo: ao mesmo tempo em que cresce, desvenda importantes fragilidades inerentes a processos de mudança. Em relação às práticas profissionais, estas não conseguem atender adequadamente as novas necessidades de prestação dos cuidados de saúde (integralidade, visão ampliada do processo saúde-doença, formação de vínculos, abordagem familiar, trabalho em equipe). O mesmo ocorre em relação à gestão do sistema (lógica quantitativa da produção, rigidez nos processos de trabalho, fixação das equipes, normatização excessiva, baixa capacidade de inovação gerencial, grande dependência dos serviços secundários, referência e contra-referência).

Nesse sentido, a Saúde da Família agudiza o debate no interior do SUS, demonstrando a fragilidade do nosso processo de construção social da saúde que tem avançado pontualmente, mediante gradativas acumulações e superposição de experiências que contemplam os enfoques das Ações Programáticas de Saúde, Vigilância da Saúde e Em Defesa da Vida e das concepções da Atenção Primária e da Atenção Básica. A nosso ver, dada a complexidade e heterogeneidade do nosso país com seus 5.564 sistemas municipais de saúde, a adoção e mesmo superposição desses diferentes referenciais é, de certa forma, esperada. Entretanto, se por 
um lado afirma as singularidades das experiências, por outro, também demonstra a inconsistência teórico-conceitual presente em nosso cenário.

Se o fundamental é potencializar esforços que impulsionem mudanças em direção à construção de um novo paradigma no setor, nos parece que o aclaramento destas questões pode resultar numa importante sinergia em torno não apenas da Saúde da Família enquanto prin-

\section{Resumo}

O objetivo deste trabalho é analisar os conceitos de Atenção Primária, Atenção Básica e Saúde da Família, com base em documentos oficiais do Ministério da Saúde, relatórios finais das Conferências Nacionais de Saúde e Conferências Nacionais de Recursos Humanos, da Norma Operacional Básica/Recursos Humanos e de textos disponibilizados on-line pela BIREME. A análise dos dados, feita mediante matrizes de dupla entrada, evidenciou a falta destes referenciais na formulação e implementação das políticas de saúde. A NOB-96 tem papel diferenciado neste aspecto; os relatórios das conferências nacionais demonstram uma lacuna importante neste debate e os artigos publicados apresentam, na maioria, os conceitos de atenção primária e atenção básica com o significado de unidade de saúde ou serviço local. Os artigos sobre Programa Saúde da Família se referem a ele mais como programa do que como estratégia, e entre os artigos que problematizam tais conceitos evidencia-se a influência das racionalidades que são subjacentes às diferentes estratégias de organização dos serviços presentes no cenário brasileiro, que são as da Vigilância à Saúde, das Ações Programáticas de Saúde e da Em Defesa da Vida.

Cuidados Primários de Saúde; Programa Saúde da Família; Política de Saúde

\section{Agradecimentos}

À Escola Nacional de Saúde Pública Sergio Arouca, Fundação Oswaldo Cruz, pela iniciativa em proporcionar aos alunos dos cursos de Mestrado e Doutorado, a Oficina de Redação de Artigos Científicos, desejando que esta iniciativa tenha continuidade. Em especial, quero agradecer a colaboração das professoras Dra. Virgínia Hortale e Dra. Sarah Escorel (orientadora) pelas contribuições ao artigo. cipal estratégia da Atenção Básica, como da reforma sanitária brasileira em si. Uma agenda pactuada entre gestores das três esferas de governo, aprofundando e qualificando os debates em torno desses referenciais teóricos pode contribuir para tornar a gestão pública em saúde comprometida com mudanças mais efetivas que atendam as necessidades de saúde da população.

\section{Referências}

1. Mattos RA. Desenvolvendo e oferecendo idéias: um estudo sobre a elaboração de propostas de política de saúde no âmbito do Banco Mundial [Tese de Doutorado]. Rio de Janeiro: Instituto de Medicina Social, Universidade do Estado do Rio de Janeiro; 2000.

2. Mattos RA. As agências internacionais e as políticas de saúde nos anos 90: um panorama geral da oferta de idéias. Ciênc Saúde Coletiva 2001; 6: 377-89.

3. Fundo das Nações Unidas para a Infância. Cuidados primários de saúde - relatório da Conferência Internacional sobre Cuidados Primários de Saúde - Alma Ata, 1978. Brasília: Fundo das Nações Unidas para a Infância; 1979.

4. Walt G. World health: globalisation of international health. Lancet 1998; 351:434.

5. Mendes EV. Uma agenda para a saúde. São Paulo: Editora Hucitec; 1996.

6. Negri B. A política de saúde no Brasil nos anos 1990: avanços e limites. In: Negri B, Viana ALD, organizadores. O Sistema Único de Saúde em dez anos de desafio. São Paulo: Sobravime; 2002. p. $15-41$

7. Souza RR. O financiamento federal do SUS: mitos e verdades. In: Negri B, Viana ALD, organizadores. O Sistema Único de Saúde em dez anos de desafio. São Paulo: Sobravime; 2002. p. 411-32.

8. Campos GWS. Reforma da reforma: repensando a saúde. São Paulo: Editora Hucitec; 1992.

9. Negri B, Werthein J. Apresentação. In: Starfield B, organizador. Atenção primária: equilíbrio entre necessidades de saúde, serviços e tecnologia. Brasília: Organização das Nações Unidas para a Educação, a Ciência e a Cultura/Ministério da Saúde; 2002. p. 13-4.

10. Escorel S. Reviravolta na saúde: origem e articulação do movimento sanitário. Rio de Janeiro: Editora Fiocruz; 1998

11. Costa NR, Pinto LF. Piso da Atenção Básica: mudanças na estrutura. In: Negri B, Viana ALD organizadores. O Sistema Único de Saúde em dez anos de desafio. São Paulo: Sobravime; 2002. p. 271-98. 
12. Ministério da Saúde. Norma Operacional Básica do Sistema Único de Saúde/NOB-SUS 93. Brasília: Ministério da Saúde; 1993.

13. Ministério da Saúde. Norma Operacional Básica do Sistema Único de Saúde/NOB-SUS 96. Brasília: Ministério da Saúde; 1997.

14. Ministério da Saúde. Regionalização da assistência à saúde: Norma Operacional da Assistência à Saúde, 2002. Brasília: Ministério da Saúde; 2002.

15. Ministério da Saúde. Legislação do SUS. Lei n. 8.080/90. http://www.saúde.gov.br/ (acessado em 13/Abr/2005).

16. Ministério da Saúde. Legislação do SUS. Lei n. 8.142/90. http://www.saúde.gov.br/ (acessado em 13/Abr/2005).

17. Coordenação de Saúde da Comunidade, Departamento de Assistência e Serviços de Saúde, Secretaria de Assistência à Saúde, Ministério da Saúde. Saúde da família no Brasil: linhas estratégicas para o quadriênio 1999/2002. Brasília: Ministério da Saúde; 1999.

18. Departamento de Atenção Básica, Secretaria de Assistência à Saúde, Ministério da Saúde. Manual para a organização da atenção básica. Brasília: Ministério da Saúde; 1999.

19. Departamento de Atenção Básica, Secretaria de Assistência à Saúde, Ministério da Saúde. O trabalho do Agente Comunitário de Saúde. Brasília: Ministério da Saúde; 2000.

20. Departamento de Atenção Básica, Secretaria de Assistência à Saúde, Ministério da Saúde. Guia prático de implantação do Programa Saúde da Família. Brasília: Ministério da Saúde; 2001.

21. Fundação Oswaldo Cruz. História da 7ạ Conferência Nacional de Saúde. http://www.fiocruz. $\mathrm{br} / \mathrm{histconferencias/setima.html} \mathrm{(acessado} \mathrm{em}$ 20/Jan/2004).

22. Conselho Nacional de Saúde. 8a Conferência Nacional de Saúde. Saúde como direito inerente à personalidade e à cidadania; reformulação do sistema nacional de saúde e financiamento setorial. Brasília: Ministério da Saúde; 1986. (Relatório final).

23. Conselho Nacional de Saúde. 9ạ Conferência Nacional de Saúde. Saúde: municipalização é o caminho. Brasília: Ministério da Saúde; 1992. (Relatório final).

24. Conselho Nacional de Saúde. 10ạ Conferência Nacional de Saúde. SUS: construindo um modelo de atenção à saúde para a qualidade de vida. Brasília: Ministério da Saúde; 1996. (Relatório final).

25. Conselho Nacional de Saúde. 11ạ Conferência Nacional de Saúde. Efetivando o SUS: acesso, qualidade e humanização na atenção à saúde, com controle social. Brasília: Ministério da Saúde; 2000. (Relatório final).

26. Conselho Nacional de Saúde. 12a Conferência Nacional de Saúde. Conferência Sergio Arouca. Saúde: um direito de todos e um dever do estado. A saúde que temos, o SUS que queremos. Brasília: Ministério da Saúde; 2003. (Relatório final).

27. Conselho Nacional de Saúde. 1ạ Conferência Nacional de Recursos Humanos em Saúde. Brasília: Ministério da Saúde; 1986. (Relatório final).
28. Conselho Nacional de Saúde. 2ạ Conferência Nacional de Nacional de Recursos Humanos em Saúde. Brasília: Ministério da Saúde; 1993. (Relatório final).

29. Conselho Nacional de Saúde, Ministério da Saúde. Princípios e diretrizes para a gestão do trabalho no SUS (NOB/RH-SUS). Brasília: Ministério da Saúde; 2005.

30. Viana ALD, Heimann LS, Lima LD, Oliveira RG, Rodrigues SH. Descentralização no SUS: efeitos da NOB-SUS 01/96. In: Negri B, Viana ALD, organizadores. O Sistema Único de Saúde em dez anos de desafio. São Paulo: Sobravime; 2002. p. 471-88.

31. Scatena JHG, Tanaka OY. A descentralização da saúde no Estado de Mato Grosso, Brasil: financiamento e modelo de atenção. Rev Panam Salud Pública 2000; 8:242-9.

32. Viana ALD, Dal Poz MR. A reforma sanitária do sistema de saúde no Brasil e o Programa de Saúde da Família. Physis 1998; 8:148.

33. Starfield B, organizador. Atenção primária: equilíbrio entre necessidades de saúde, serviços e tecnologia. Brasília: Organização das Nações Unidas para a Educação, a Ciência e a Cultura/Ministério da Saúde; 2002.

34. Campos FE, Belisário SA. O Programa de Saúde da Família e os desafios para a formação profissional e a educação continuada. Interface Comun Saúde Educ 2001; 5:133-42.

35. Conill EM. Políticas de atenção primária e reformas sanitárias: discutindo a avaliação a partir da análise do Programa Saúde da Família em Florianópolis, Santa Catarina, Brasil, 1994-2000. Cad Saúde Pública, 2002; 18 Suppl:191-202.

36. Marques RM, Mendes A. A política de incentivos do Ministério da Saúde para a atenção básica: uma ameaça à autonomia dos gestores municipais e ao princípio da integralidade? Cad Saúde Pública 2002; 18 Suppl:163-71.

37. Fernandes JCL. Agentes de saúde em comunidades urbanas. Cad Saúde Pública 1992; 8:134-9.

38. Merhy EE, Franco TB. Programa Saúde da Família: somos contra ou a favor? Saúde Debate 1999; 26:118-22.

39. Merhy EE. E daí surge o PSF como uma continuidade e um aperfeiçoamento do PACS. Interface Comun Saúde Educ 2001; 9:147-9.

40. Testa M. Pensar em saúde. Porto Alegre: Artes Médicas; 1992.

41. Schraiber LB, organizador. Programação em saúde hoje. São Paulo: Editora Hucitec; 1990.

42. Carvalho SR, Campos GWS. Modelos de atenção à saúde: a organização de Equipes de Referência na rede básica da Secretaria Municipal de Saúde de Betim, Minas Gerais. Cad Saúde Pública 2000; 16:507-15.

43. Campos GWS. A saúde pública e a defesa da vida. 2a Ed. São Paulo: Editora Hucitec; 1992.

44. Teixeira CF. A mudança do modelo de atenção à saúde no SUS: desatando nós, criando laços. Saúde Debate 2003; 27:257-77.

Recebido em 31/Mai/2005

Versão final reapresentada em 06/Out/2005 Aprovado em 10/Out/2005 